\title{
MICHEL BAKOUNINE
}

\author{
THEORIE ET PRATIQUE DU FEDERALISME ANTI-ETATIQUE \\ EN 1870-1871
}

Lorsque la guerre franco-allemande éclata, Michel Bakounine, rentré en Europe le 27 décembre 1861 après onze ans de prison et d'exil, avait, depuis cinq ou six années, donné une forme définitive aux idées qui sont restées pour toujours associées à son nom.

Ces idées forment un tout cohérent ${ }^{\mathbf{1}}$ : une philosophie sociale à laquelle sa pratique révolutionnaire était étroitement liée. Aussi, très tôt après son retour, Bakounine avait-il repris ses activités révolutionnaires et, dès 1863, il était d'avis qu'il fallait prendre garde de ne pas être pris au dépourvu par le bouleversement à venir. C'est pour faire face à une telle situation qu'il commençait à organiser des militants sur le plan international, bien avant la fondation de la Première Internationale, afin d'agir sur ces événements en temps utile et d'éviter ainsi les erreurs des révolutions de 1848-1849, afin de contrarier surtout le courant "néojacobin». La tradition maçonnique et carbonariste, les activités souterraines de Buonarroti pendant quarante ans, les organisations secrètes néo-babouvistes, buonarrotistes des années 30 , celles de Mazzini, des Polonais, de Marx et Engels, en un mot, toute l'histoire clandestine du mouvement social international de 1820 jusqu'à 1850 , voire après, ont incité Bakounine, qui avait pu en avoir souvent une connaissance intime, lors de ses séjours en Suisse, en France, en Belgique et en Allemagne en 1840-1849, à donner à ces idées la forme de statuts et programmes destinés à des sociétés secrètes, dont la première fut ébauchée en 1864, la dernière en 1872 - organisations qui étaient, d'ailleurs, éphémères ou même non-existantes. ${ }^{2}$

Il y a néanmoins deux aspects qu'il faut retenir de ces organisations "fantômes". D'abord, sa véritable manie d'écrire des programmes, statuts, règlements a amené Bakounine à fixer l'essentiel de ses idées dans ces documents, ce qui, d'ailleurs, n'a pas été tellement favorable à

1 Contrairement à ce qu'on pourrait penser à cause de la forme circonstancielle et peu systématique sous laquelle elles sont présentées, à. l'époque comme après. 2 La plupart de ces programmes n'étaient que des projets, dont plusieurs sont encore inédits. 
leur propagation. On doit ensuite considérer l'importance que Bakounine attachait à ces noyaux ou «nuclei», pour ses activités et sa tactique avant et pendant la Révolution. C'est ainsi que, le $1^{\text {er }}$ avril 1870 , il écrivait à l'International lyonnais Albert Richard:

"Les révolutionnaires politiques, les partisans de la dictature ostensible, une fois la révolution ayant obtenu un premier triomphe, recommandent l'apaisement des passions, l'ordre, la confiance et la soumission aux pouvoirs révolutionnairement établis; de cette manière, ils reconstituent l'Etat. Nous, au contraire, nous devrons fomenter, éveiller, déchaîner toutes les passions, nous devons produire l'anarchie et, pilotes invisibles au milieu de la tempête populaire, nous devrons la diriger, non par un pouvoir ostensible quelconque, mais par la dictature collective de tous les alliés - dictature sans écharpe, sans titre, sans droit officiel, et d'autant plus puissante qu'elle n'aura aucune des apparences du pouvoir. Voici la seule dictature que j'admette. Mais pour qu'elle puisse agir, il faut qu'elle existe et, pour cela, il faut la préparer et l'organiser d'avance; car elle ne se fera pas toute seule, ni par des discussions, ni par des expositions et débats de principe, ni par des assemblées populaires. Peu d'alliés, mais bons, mais énergiques, mais discrets, mais fidèles, mais surtout libres de vanité et d'ambition personnelles, des hommes forts, assez sérieux, ayant l'esprit et le cœur assez haut placés pour préférer la réalité de la force à ses apparences vaniteuses.» ${ }^{1}$

Deux mois plus tard, Bakounine s'adressait dans le même sens à Sergej Nečaev. ${ }^{2}$

En ce qui concerne les idées de Bakounine, tout est bien clair: l'essentiel en est connu depuis 1900, depuis la publication de la grande biographie que lui consacra Max Nettlau. ${ }^{3}$ Nous ajoutons que, en outre,

1 Bibliothèque de la Ville de Lyon, ms. 5401/12.

2 Archives Bakounine, t. IV, Michel Bakounine et ses relations avec Sergej Nečaev, 1870-1872, Leyde 1971, pp. 230-231, 236-238. De même, dans sa Lettre à un Français, Bakounine écrit: "Que doivent donc faire les autorités révolutionnaires - et tâchons qu'il y en ait aussi peu que possible - que doivent-elles faire pour étendre et pour organiser la révolution? Elles doivent non la faire elles-mêmes par des décrets, non l'imposer aux masses, mais la provoquer dans les masses. Elles doivent non leur imposer une organisation quelconque, mais en suscitant leur organisation autonome de bas en haut, travailler sous main, à l'aide de l'influence individuelle sur les individus les plus intelligents et les plus influents de chaque localité, pour que cette organisation soit autant que possible conforme à nos principes. Tout le secret de notre triomphe est là.) (Michel Bakounine, CEuvres, t. II, Paris 1907, p. 228)

3 Max Nettlau, Michael Bakunin. Eine Biographie (The Life of Michael Bakounine), 3 vol., Londres 1896-1900. 
pour l'époque qui nous intéresse ici, de juillet 1870 à mai 1871 , tous ses écrits ont été publiés à titre posthume, à l'exception de deux seules brochures. ${ }^{1}$ A quel point ces idées ont-elles influencé ses disciples et, en général, les militants de l'Internationale dont Bakounine était membre depuis l'été 1868? Question complexe, à laquelle il n'est pas toujours facile de répondre. Sur bien des points, nous sommes suffisamment informés; sur d'autres, nombre de documents sont perdus, et ils le sont pour toujours. De surcroît, l'histoire exacte du mouvement social de cette époque cruciale est encore à écrire. Cependant, les documents disponibles permettent de tracer, à grands traits, les opinions de $\mathbf{B a}-$ kounine sur les événements politiques, les moyens qu'il préconisait, la tactique qu'il voulait suivre, et ses propres activités.

En 1866, dans un long manuscrit qu'on connaît sous le titre de Catéchisme révolutionnaire, Bakounine exposait son programme politique et social. La base de toute organisation politique d'un pays doit être la commune absolument autonome, qui nomme par élection et destitue, s'il le faut, tous ses fonctionnaires, qui a le droit incontestable de créer sa propre législation, de se donner sa propre constitution. La province ne doit être qu'une fédération libre de communes autonomes; la nation ne sera qu'une fédération de provinces autonomes. Evitant la concentration de toutes les forces révolutionnaires d'un pays en un seul point, la révolution une pourra jamais réussir que si elle entraîne à la fois toutes les masses des campagnes aussi bien que des villes». ${ }^{2}$

Deux ans plus tard, en 1868, Bakounine parle à nouveau de la Commune qui remplacera l'Etat, et c'est alors qu'on trouve pour la première fois sous sa plume l'expression de «fédération des barricades». La Commune sera une fédération des barricades en permanence, un Conseil composé par la délégation "d'un ou deux députés par chaque barricade, un par rue, ou par quartier, députés investis de mandats impératifs, toujours responsables et toujours révocables». ${ }^{3}$ En d'autres mots, un soviet de députés du peuple en armes.

Dès la fin des années 60, Bakounine attendait la chute du Second Empire et faisait rayonner son inlassable activité dans plusieurs

1 Ne furent publiées que les Lettres à un Français sur la crise actuelle, s.1., septembre 1870,43 pp., dont le texte a été rédigé, en partie remanié, par James Guillaume: "raccourci, pour ne point dire châtré», d'après Bakounine; et L'Empire knouto-germanique et la révolution sociale, Première livraison, Genève 1871, 136 pp. Tous les autres manuscrits de cette époque ont été publiés en 1907, 1908 en 1910, dans les Euvres; ils ne sont pas encore complètement édités.

2 Nettlau, op. cit., pp. 218, 225-226.

3 L'Alliance de la Démocratie socialiste et l'Association internationale des Travailleurs, Rapport et documents publiés par ordre du Congrès international de La Haye, Londres 1873, p. 130. 
directions à la fois. Il espérait qu'une révolution en France influerait sur le mouvement révolutionnaire en Espagne et en Italie, pourrait, gagnant par le relais des peuples slaves d'Autriche la Pologne et l'Ukraine, s'étendre jusqu'aux masses paysannes russes. ${ }^{1}$ En France, épicentre du bouleversement, il tablait non pas d'abord sur une révolution à Paris, mais en province, dans le Sud-Est, à Marseille, à Lyon surtout, la deuxième ville de France, Lyon, la traditionnellement révolutionnaire. Dans cette "capitale du socialisme», l'Internationale s'était solidement implantée, les idées de Bakounine profondément enracinées.

C'était à Lyon qu'avait eu lieu, le 13 mars 1870, la manifestation la plus caractérisée de l'A.I.T., présidée par Eugène Varlin. Invité, Bakounine chargea Adhémar Schwitzguébel (qui, dès 1868, faisait partie de son cercle (intime») d'apporter, en tant que représentant de la Fédération jurassienne, un message qui devait être lu à ses amis. Deux textes (non datés) de cette époque forment les parties substantielles d'un programme d'action dont l'essentiel était que «les communes révolutionnaires de Paris, Lyon, Marseille, Lille, Bordeaux, Rouen, Nantes etc., ayant solidairement et uniformément préparé et dirigé le mouvement révolutionnaire pour le renversement de la tyrannie impériale, déclarent qu'elles ne considéreront leur tâche comme achevée que quand elles auront assuré le triomphe de la révolution par l'application des principes égalitaires qu'elles professent. $\rangle^{2}$

Quelques semaines après l'assemblée de Lyon, Bakounine écrit à Albert Richard une lettre où il expose son programme et critique les idées "jacobines» de ce dernier. Selon Richard, estimait-il, la révolution ayant éclaté à Paris, les grandes villes y enverraient leurs délégués pour former une sorte de Comité de Salut public qui décréterait la révolution, l'abolition du vieil Etat, la liquidation sociale, la propriété collective, et organiserait un Etat révolutionnaire suffisamment fort pour réprimer la réaction intérieure et extérieure. Bakounine se dit d'un avis tout à fait opposé à celui qu'il croit voir affirmer par Richard: même si c'est Paris qui commence la révolution - ce qu'il n'estime pas du tout certain -, sa tâche sera seulement de détruire et liquider l'Etat et d'en proclamer la banqueroute totale. Bien sûr, Paris s'organisera en formant la fédération révolutionnaire de tous les quartiers, la "commune fédérative», mais il renoncera immédiatement au droit de gouverner

1 Max Nettlau, Der Anarchismus von Proudhon zu Kropotkin. Seine historische Entwicklung in den Jahren 1859-1880, Berlin 1927, p. 120.

2 Probablement écrit entre le 15 et le 26 septembre 1870, Pis'ma M. A. Bakunina k A. I. Gercenu i N. P. Ogarevu, Genève 1896, p. 307. En dehors de ce texte, il existe encore un autre sous le titre "Mesures précédant la révolution et la préparant», ibid., p. 309. 
et d'organiser la France. De même, l'Assemblée révolutionnaire fédérale des délégués des provinces et des communes, qui se soulèveront simultanément, et indépendamment, n'aura pas pour tâche d'organiser la France, mais devra être l'expression d'une organisation non-étatique spontanée. ${ }^{1}$

Déjà, à la mi-juillet, Bakounine estimait que la guerre francoprussienne était inévitable, et inévitable la défaite de la France bonapartiste. Cette défaite ne pouvait mener qu'à la révolution sociale et il fallait que les révolutionnaires, ceux surtout de l'Internationale, fussent prêts à prendre la part la plus active aux événements qui n'allaient pas tarder à se produire. ${ }^{2}$ Dès la première nouvelle des succès allemands, il redoubla fiévreusement d'activité. En trois jours, il écrivait vingt-trois longues lettres et commença sa Lettre à un Français. Deux idées fondamentales sont à retenir de cet écrit. Bakounine prévoyait la création d'un Gouvernement de Défense nationale qui «n'aura qu'un programme: maintenir l'ordre». Il fallait déchaîner une révolution sociale, le salut de la France dépendant d'un soulèvement général du peuple, mais, et la vue est neuve dans une France dominée, accablée par le poids de sa capitale, "ce ne sera pas Paris qui pourra prendre cette fois l'initiative de la vraie révolution - l'initiative appartiendra aux provinces». ${ }^{3}$

Deux jours avant Sedan, il écrivait encore: "Paris, absorbé par l'unique intérêt et par la seule pensée de sa défense, sera tout à fait incapable de diriger et d'organiser le mouvement national de la France. S'il pouvait avoir cette prétention saugrenue, ridicule, il tuerait le mouvement, et il serait par conséquent du devoir de la France, des provinces, de lui désobéir, dans l'intérêt suprême du salut national. La seule et meilleure chose que Paris puisse faire dans celui de son propre salut, c'est de proclamer et de provoquer l'absolue indépendance et spontanéité des mouvements provinciaux; et si Paris oublie ou néglige de le faire, par quelque raison que ce soit, le patriotisme commande aux provinces de se lever et de s'organiser spontanément, indépendamment

1 Lettre du 1 er avril 1870, Bibliothèque de la Ville de Lyon, ms. 5401/12.

2 L'opinion de Bakounine, selon Arman Ross, était que «la France bonapartiste serait vaincue, l'Empire étant gangrené et en pleine décomposition, alors que la Prusse était un puissant Etat militaire. La défaite de la France déclencherait la révolution sociale et les révolutionnaires, l'Internationale en tête, devaient se tenir prêts à prendre une part des plus actives aux événements qui n'allaient pas manquer de se produire." (M. P. Sažin, "Vospominanija o M. A. Bakunine», in: Michailu Bakuninu, 1876-1926. Očerki istorii anarchičeskogo dviženija v Rossii, Moscou 1926, p. 176). (Sauf indication contraire, les citations traduites sont empruntées aux travaux préparatoires pour les Archives Bakounine. Réd.)

3 La Solidarité. Organe des sections de la Fédération romande de l'A.I.T., Neuchâtel, nº 20,20 août 1870 , p. 2. 
de Paris, pour le salut de la France et de Paris lui-même. Il résulte de tout cela, d'une manière évidente, que si la France peut encore être sauvée, ce n'est que par le soulèvement spontané des provinces."1

Ses opinions ne changèrent pas après le 4 septembre. Au contraire, Bakounine était convaincu qu'il fallait absolument continuer la guerre contre les envahisseurs, mais, qu'en même temps, il était nécessaire de faire la révolution contre le régime républicain bourgeois, et, disait-il, paraphrasant les mots de Danton en 1792, «avant de marcher contre l'ennemi, il faut le détruire, le paralyser derrière soi»: "Il faut mettre à bas les Prussiens de l'intérieur pour pouvoir marcher ensuite avec confiance et sécurité contre les Prussiens de l'extérieur.»" Il eût voulu empêcher la consolidation du gouvernement républicain et, la guerre patriotique continuant, avait mis son espoir dans un renforcement simultané de l'élan révolutionnaire, seule possibilité à ses yeux de gagner la population des campagnes à la révolution. Comprenant très bien que seuls les ouvriers urbains se trouveraient du côté de la Révolution, Bakounine était d'avis qu'une révolution dans les villes entraînerait les paysans, que ce n'était qu'à cette condition que la révolution sociale serait possible et que la France pourrait être sauvée.

Il est évident que Bakounine fut seul, parmi les révolutionnaires de l'Internationale, à envisager ainsi la situation politique et les perspectives révolutionnaires. Ceci par exemple à l'inverse d'Auguste Blanqui ou des «néo-jacobins», dont l'opinion était bien exprimée par le mot d'ordre de Blanqui, "En présence de l'ennemi, plus de partis ni de nuances», et qui, jusqu'en octobre, manifestaient l'intention de collaborer avec le gouvernement de Défense nationale. Pour eux, la question sociale avait cédé le pas à la question nationale. Les opinions de Bakounine se basaient sur une appréciation correcte de la situation: la défaite n'avait pas seulement résulté dans la chute du régime, mais encore affaibli, voire désintégré l'appareil militaire et, par là, l'Etat. Par conséquent, un des éléments essentiels pour la révolution était là. Bakounine rejetait toute "union sacrée», toute trêve avec le gouvernement qu'il considérait, dès l'instant, comme contre-révolutionnaire. C'est pour défendre leurs domination et intérêts de classe que les classes dirigeantes se font les complices des envahisseurs. Toute la politique et l'action du gouvernement de Défense nationale étaient dominées par la pensée de "se défaire de la révolution». ${ }^{3}$ Toute la

1 Bakounine, Euvres, t. II, p. 214.

2 Lettre du 23 août 1870 à Albert Richard, Bibliothèque de la Ville de Lyon, ms. 5401/17.

3 Edouard Dolleans, dans l'introduction au livre de Maurice Dommanget, Blanqui, la guerre de 1870-1871 et la Commune, Paris 1947, p. VI. 
différence entre l'attitude de Bakounine et celle de Blanqui réside dans ce fait que, pour le premier, la situation désastreuse de la France commandait à déchaîner la révolution sociale au lieu de la mettre en veilleuse. $^{1}$

Pour Marx aussi, le ralliement au gouvernement du 4 Septembre s'imposait. Dans le Second Manifeste sur la guerre (du 9 septembre 1870), il écrit, au nom du Conseil général de l'A.I.T.: "Toute tentative de renverser le nouveau gouvernement, quand l'ennemi frappe presque aux portes de Paris, serait une folie désespérée. Les ouvriers français doivent remplir leur devoir de citoyens; mais en même temps ils ne doivent pas se laisser entraîner par les souvenirs nationaux du Premier Empire. [...] Que calmement et résolument, ils profitent de la liberté républicaine pour procéder méthodiquement à leur propre organisation de classe. $\|^{2}$ Dans le même sens, Eugène Dupont, correspondant pour la France du Conseil général, avait envoyé de Londres des instructions aux correspondants français de l'Internationale, insistant sur la nécessité de ne pas continuer la guerre mais de «laisser cette vermine bourgeoise faire la paix avec les Prussiens et de profiter des libertés que les circonstances vont apporter pour organiser toutes les forces de la classe ouvrière». ${ }^{3}$ De même, Auguste Serraillier fut délégué à Paris par le Conseil général, pour y contrarier toute tentative de soulèvement. ${ }^{4}$

Il semble bien que le Conseil fédéral parisien, quoique ne s'opposant pas au courant patriotique et insistant sur le soutien à la défense nationale, ait pris une attitude plus réservée. Dans sa circulaire aux Internationaux des provinces, il préconisa la surexcitation, par tous les moyens possibles, du patriotisme qui devait sauver la France révolutionnaire, et recommanda de prendre des mesures énergiques contre la

1 Maurice Dommanget note que, le 14 août, il existait une situation révolutionnaire. Commentant les opinions de Blanqui concernant le soulèvement de La Villette, il écrit: "Une fois de plus [Blanqui] retombe dans l'insurrection conçue suivant les vieilles méthodes, une fois de plus il confond le coup de main isolé d'une petite troupe à date et à heure fixes avec la Révolution d'un peuple entier, surgie du consentement de tous, à la suite d'un événement sensationnel, Révolution qui peut réussir plus facilement et plus vite si un parti ou une société secrète en prend la direction, mais qui n'en est pas moins marquée inexorablement au cadran de l'histoire." (Dommanget, op. cit., p. 20)

2 La Guerre civile en France, 1871, Paris 1953, p. 289. Dans le même sens, Engels écrit à Marx, le 7 septembre 1870: "Il faut utiliser les libertés consenties inévitablement par la République pour organiser le parti en France [...]; réserver l'Internationale en France jusqu'à ce que la paix soit conclue." (Karl Marx et Friedrich Engels, Werke, t. 33, Berlin 1966, p. 57)

3 Oscar Testut, L'Internationale et le jacobinisme au ban de l'Europe, t. II, Paris 1872, p. 26.

- Voir The General Council of the First International, 1870-1871, Minutes, [t. IV,] pp. 139-144. 
réaction bourgeoise et bonapartiste et de pousser à l'acceptation des grandes mesures de défense par l'organisation des comités républicains, premiers éléments des futures communes révolutionnaires. Néanmoins, il ajoutait: "Notre révolution à nous n'est pas encore faite, et nous la ferons lorsque, débarrassés de l'invasion, nous jetterons révolutionnairement les fondements de la société égalitaire que nous voulons.» ${ }^{1}$ En d'autres termes, d'abord la guerre, et ensuite la révolution.

Parmi les signataires du manifeste figurait Varlin qui, il y avait quelques mois, au Congrès de Bâle, avait défendu le collectivisme et dès ce moment, appartenait au groupe intime de Bakounine. Le 25 décembre 1869, Varlin avait écrit qu'il fallait aux Internationaux encore un an, deux ans peut-être, de propagande et d'organisation pour faire la révolution qui, outre la France, engloberait l'Espagne et l'Italie, et signifierait donc une révolution sociale européenne. ${ }^{2}$

Ces mots trouvèrent leur confirmation lorsque, le 30 août $1870, \mathrm{Ba}-$ kounine écrivit: «Il y a encore un an, nous nous préparions à la révolution que nous attendions, les uns plus tôt, les autres plus tard; et maintenant, quoi qu'en disent les aveugles, nous sommes en pleine révolu-

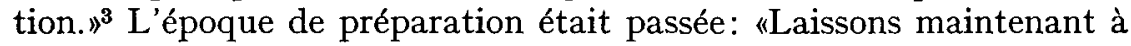
d'autres le soin de développer théoriquement les principes de la révolution sociale, et contentons-nous de les appliquer largement, de les incarner dans les faits." "Maintenant, dit-il, nous devons nous embarquer tous ensemble sur l'océan révolutionnaire, et désormais nous devons propager nos principes non plus par des paroles, mais par des faits, car c'est la plus populaire, la plus puissante et la plus irrésistible des propagandes. Taisons quelquefois nos principes quand la politique, c'est-à-dire quand notre impuissance momentanée vis-à-vis d'une grande puissance contraire l'exigera, mais soyons toujours impitoyablement conséquents dans les faits. Tout le salut de la révolution est là.»" Comme l'a très justement remarqué Maurice Dommanget, Bakounine, à l'opposé de Blanqui - et, ajouterons-nous, de Marx -, "se maintint dur comme fer sur le plan révolutionnaire et, plus que Blanqui, analysa les événements du point de vue de la lutte des classes. $\eta^{5}$

1 Cette circulaire fut imprimée, par erreur, comme affiche, cf. l'article de G. Langkau ci-dessus, p. 135, note 2 .

2 Lettre à Aubry du 25 décembre 1869, publiée dans le Troisième Procès de 1'Association internationale des Travailleurs à Paris, Paris, juillet 1870, p. 35.

3 Bakounine, Euvres, t. II, pp. 226-227. En général, Bakounine était d'opinion qu' ‘il y a des périodes dans l'histoire où les révolutions sont tout bonnement impossibles; il en est d'autres où celles-ci sont inéluctables" (Archives Bakounine, t. IV, p. 230).

4 Bakounine, Euvres, t. II, p. 227.

5 Dommanget, op. cit., p. 51 . 
Le 4 septembre 1870, Bakounine se met à la disposition des Lyonnais. Dans une lettre à Lyon datée de ce jour-là, il expose la tactique révolutionnaire à suivre. Le peuple français, écrit-il, ne doit plus compter sur aucun gouvernement, ni existant ni même révolutionnaire. $\mathrm{La}$ machine gouvernementale étant brisée, le peuple doit se lever et s'organiser de bas en haut, en dehors de toute direction officielle, en déclarant partout la déchéance de l'Etat. Il ne doit laisser debout qu'une seule loi, celle du salut de la France contre les Prussiens à l'extérieur, contre les traîtres à l'intérieur. En arrachant les armes à ceux qui les détiennent, toutes les communes doivent envoyer leurs délégués en un lieu quelconque en dehors de Paris, pour former un gouvernement provisoire. Il faut que les grands centres provinciaux, Lyon et Marseille, prennent cette initiative, et la prennent immédiatement. Sinon, la France et le socialisme européen sont perdus. "L'hésitation serait donc un crime. ${ }^{1}$ La proclamation du 8 septembre de l'Internationale lyonnaise était sans dout basée sur cette lettre. ${ }^{2}$

Le 15 septembre, Bakounine arrive à Lyon, accompagné de ses deux amis l'ex-officier russe Vladimir Ozerov et le jeune Polonais Valence Lankiewicz, qui sera tué aux avant-postes de la Commune de Paris. Le 17 septembre est fondé le Comité de Salut de la France qui, le 26, organise un meeting où fut lu le fameux appel de la Fédération révolutionnaire des communes, rédigé la veille par Bakounine et placardé sur les murs de Lyon le jour suivant. Il y était dit entre autres: "Toutes les organisations municipales existantes sont cassées et remplacées dans toutes les communes fédérées par des Comités de Salut de la France qui exerceront tout le pouvoir sous le contrôle immédiat du peuple.» ${ }^{3}$ Il ne s'agit pas de reprendre ici l'histoire de ce soulèvement dont l'échec est connu. Ces proclamations furent cependant les premières protestations publiques contre l'Etat qui, quelques mois plus tard, trouveront dans le programme de la Commune de Paris leur signification historique.

On a beaucoup critiqué ce soulèvement manqué ainsi que l'activité de Bakounine. Pour Marx, Bakounine était un «âne» (pas le seul, d'ailleurs, dans le bestiaire épistolaire de Marx et Engels). "On n'aurait pas pu mieux faire aux bureaux de la presse à Berlin pour servir Bismarck", écrit le Volksstaat. ${ }^{4}$ Marx faisait encore la critique du fait que Bakou-

1 Lettre à Albert Richard du 4 septembre 1870, datée, par erreur, du 4 août, Bibliothèque de la Ville de Lyon, ms. 5401/14.

2 Voir Antoine Olivesi, La Commune de 1871 à Marseille et ses origines, Paris 1950, pp. 93-94.

3 Testut, op. cit., p. 40.

4 Numéro du 12 octobre 1870. Dans sa lettre Aux compagnons de la Fédération jurassienne (février-mars 1872), Bakounine écrit qu' «enfin, au mois de mars 
nine, étant russe, avait signé le manifeste. ${ }^{1}$ Tout le monde a exalté la participation, quelques mois plus tard, des étrangers aux événements de la Commune de Paris.

On peut bien arguer que l'effort de déchaîner la révolution à Lyon - au moment des élections de la municipalité - était venu trop tôt et n'avait pas beaucoup de chances d'aboutir. L'historien trouve toujours des arguments pour prouver que l'histoire ne pouvait pas manquer de suivre le cours qu'elle a pris, et que c'est toujours chose précaire que fixer une date à la révolution. En général, c'est trop tôt ou trop tard. Dans ce cas, on a tout lieu de penser que c'était trop tard et que, s'il y avait eu en un moment des possibilités, c'était lors de la chute de Napoléon III. C'est à bon droit que Dubreuilh remarquait: "La Commune a surgi six mois trop tard.» ${ }^{2}$ De son côté, Trotsky écrivait en 1921: "La Commune est venue trop tard. Elle avait toutes les possibilités de prendre le pouvoir le 4 septembre, et cela aurait permis au prolétariat de Paris de se mettre d'un seul coup à la tête des travailleurs du pays dans leur lutte contre toutes les forces du passé, contre Bismarck aussi bien que contre Thiers." ${ }^{3}$

Il y a eu, à l'automne 1870, une première vague révolutionnaire où Paris n'a pas joué le premier rôle. La Commune a eu un début d'existence en province, à Marseille et Lyon notamment, en septembre. On a vu s'esquisser dans le Midi et le Sud-Ouest des ligues qui réunissent déjà les caractéristiques essentielles de ce que sera la Commune de Paris. Si la guerre est alors au premier plan de leurs préoccupations, il s'agit d'une guerre révolutionnaire. Au printemps de 1871, la révolution communaliste sera leur unique souci. En mars, Paris ne commence donc pas la révolution, il la recommence; mais elle vient trop tard pour la province. ${ }^{4}$

1871, [les démocrates socialistes de l'Allemagne] durent ouvrir les yeux, et, forcés d'opter entre la révolution populaire et foncièrement socialiste de la Commune de Paris et la réaction bourgeoise représentée par Versailles, ils prirent ouvertement le parti de la première, réhabilitant par là même les insurrections précédentes de Lyon et de Marseille, qu'ils avaient d'abord si sévèrement condamnées" (Archives Bakounine, t. II, Michel Bakounine et les conflits dans l'Internationale, 1872, Leyde 1965, p. 81).

1 Lettre de Marx à Beesly du 19 octobre 1870, Marx et Engels, Werke, t. 33, p. 158.

2 Louis Dubreuilh, "La Commune», in: Jean Jaurès, Histoire socialiste (17891900), t. XI, Paris s.d., p. 249.

3 "Les leçons de la Commune», in: C. Talès, La Commune de 1871, Paris 1971, p. 167.

4 Nous reprenons les mots de Jeanne Gaillard dans Communes de province, Commune de Paris, 1870-1871, Paris 1971, pp. 137-139. 
Bakounine lui-même a bien expliqué ce qu'il avait en vue: «Nous avions voulu renverser la municipalité de Lyon $[\ldots]$, renverser en même temps tous les pouvoirs officiels $[\ldots]$ et convoquer la Convention nationale du salut de la France." ${ }^{1} \mathrm{~A}$ la suite des événements où il fut arrêté, puis délivré, Bakounine abandonna Lyon et demeura quelque temps à Marseille, recherché par les autorités de Lyon et de Tours. Il se rendit ensuite en Suisse, passant par Gênes. Son plan initial d'aller à Barcelone fut abandonné, on ne sait pas pourquoi.

Le 29 septembre, dans une disposition pessimiste, il écrit, de Lyon encore, à Louis Palix: "Le mouvement d'hier, s'il s'était maintenu triomphant, $[\ldots]$ en remplaçant la municipalité lyonnaise, à moitié réactionnaire et à moitié incapable, par un comité révolutionnaire émanant directement de la volonté du peuple, ce mouvement aurait pu sauver Lyon et la France. [...] Je quitte Lyon, cher ami, le cœur plein de tristesse et de prévisions sombres. Je commence à penser maintenant que c'en est fait de la France. Elle deviendra une vice-royauté de l'Allemagne. A la place de son socialisme vivant et réel, nous aurons le socialisme doctrinaire des Allemands, qui ne diront plus que ce que les baïonnettes prussiennes leur permettront de dire. L'intelligence bureaucratique et militaire de la Prusse unie au knout du tsar de SaintPétersbourg vont assurer la tranquillité et l'ordre public, au moins pour cinquante ans, sur tout le continent de l'Europe.»" Le 23 octobre, écrivant à Sentiñon, il reprit ces mots en ajoutant: "Adieu tous nos rêves d'émancipation prochaine. Ce sera une réaction assommante et terrible. $\|^{3}$

Si tant est que Bakounine se soit trompé sur les possibilités révolutionnaires à Lyon, il nous semble que dans sa vision, en ce qui concerne la situation politique et la tactique à suivre, il n'y avait rien d'absurde ni d'aventureux. Au contraire, son analyse politique était des plus

1 Lettre à Esquiros, 20 octobre 1870, in: Bakounine, Euvres, t. IV, Paris 1910, p. 240 .

${ }_{2}$ Testut, op. cit., p. 281. Cependant, le même jour, il écrit encore: "Outre le soin de sa propre défense, [Lyon] a donc un double devoir à remplir: celui d'organiser le soulèvement armé du Midi et celui de délivrer Paris. Il pouvait faire, il peut encore faire l'un et l'autre." (Bakounine, CEuvres, t. II, p. 289) Le 8 octobre, écrivant à Emilio Bellerio, il dit à propos de la guerre: "C'est une guerre à mort entre la révolution populaire, non bourgeoise - il n'y a plus de révolution bourgeoise, ces deux mots désormais s'excluent - et le despotisme militaire, bureaucratique et monarchique qui triomphe aujourd'hui en Allemagne." Là aussi, il nourrit encore de l'espoir: "Les amis, devenus plus prudents, plus pratiques, travaillent activement à Lyon comme à Marseille, et nous aurons bientôt notre revanche, à la barbe des Prussiens." (Nettlau, Michael Bakunin, note 4038) 3 James Guillaume, L'Internationale. Documents et souvenirs (1864-1878), t. II, Paris 1907, p. 112. 
réalistes. ${ }^{1}$ Le 4 septembre, de leur propre chef et avant Paris, Lyon et Marseille avaient proclamé la République. Le 18 septembre, à Marseille, sous la présidence d'Alphonse Esquiros, les représentants de quinze départements avaient fondé en plein accord avec l'Internationale marseillaise la Ligue du Midi. Son but était de continuer la guerre en levant une armée populaire et de combattre le gouvernement de Défense nationale. Après le 18 mars 1871, le mouvement communaliste se poursuit, notamment à Marseille, qui eut pendant treize jours sa Commune révolutionnaire, ainsi qu'à Saint-Etienne, Narbonne, Limoges. C'était le seul moyen qui aurait pu sauver la Commune de Paris, car elle était perdue dès l'instant où Thiers était allé s'installer à Versailles dans le dessein de reconquérir Paris de l'extérieur, comme Windischgrätz l'avait fait à Vienne, en octobre 1848. Dans les premiers jours de la Commune, Varlin disait à fort bon droit que, plutôt que de venir à Paris, la meilleure aide pour les Parisiens serait de soulever les provinces. $^{2}$

Après que Paris, le 18 mars 1871, eut proclamé son autonomie, on n'entendait parler que de ville libre et de commune indépendante. Le 12 avril, Pierre Vésinier écrivit: «Paris libre, c'est la Commune de Paris. La France libre, c'est la France communale fédéralisée. $\aleph^{3}$ Et dans toutes les proclamations se retrouvaient les principes communalistes et fédéralistes. Un des premiers manifestes émanant du Comité des Vingt Arrondissements et publié dans Le Cri du Peuple du 27 mars déterminait les principes généraux de l'idée révolutionnaire et fédéraliste. La Commune étant la base de tout Etat politique, elle doit être autonome, c'est-à-dire se gouverner et s'administrer elle-même. "Pour s'assurer le développement économique le plus large, l'indépendance nationale et territoriale, elle peut et doit s'associer, c'est-à-dire se fédérer avec toutes les autres communes ou associations de communes qui composent la nation. $\rangle^{4}$

Un des premiers projets présenté, le 29 mars, lors de la deuxième séance de la Commune, et rédigé entre autres par Lefrançais et Vallès,

1 En tout cas, on peut difficilement souscrire à ce qu'a écrit M. Maurice Moissonnier: "Aucune analyse de la réalité concrète et des forces politiques et sociales en présence, le mépris à l'endroit de la lutte quotidienne et des alliances nécessaires, la phrase anarchiste délirante, voilà ce qui caractérise l'influence bakouninienne!" ("La Première Internationale et la Commune à Lyon», in: La Nouvelle Critique, Paris, no 159 (octobre 1964), p. 42).

2 Communication de Nikolaj Žukovskij à Max Nettlau, in: Nettlau, Michael Bakunin, p. 546.

3 Jacques Rougerie, Paris libre 1871, Paris 1971, p. 150.

4 Jean Dautry et Lucien Scheler, Le Comité central républicain des Vingt Arrondissements de Paris (septembre 1870 - mai 1871), Paris 1960, pp. 235-239. 
allait dans le même sens et indiquait le rôle que la Commune devait s'attribuer: «Nous commençons par déclarer que la Commune de Paris est toute prête à faire un pacte d'alliance avec toute commune qui nous enverra son adhésion, et que nous hâterons de tous nos efforts la constitution de la fédération des communes de France. $)^{1}$ Le projet n'obtint pas l'approbation de tous les membres de la Commune, mais le même principe trouva son expression achevée dans la Déclaration au Peuple français du 19 avril où il est fait état de l'sautonomie absolue de la Commune étendue à toutes les localités de la France", autonomie qui n'aura pour limites que le droit d'autonomie égal pour toutes les autres communes. L'ancienne unité française n'était que la centralisation despotique; l'unité politique, telle que la veut Paris, c'est l'association volontaire de toutes les initiatives locales. En fait, cette déclaration reprenait beaucoup des idées que Bakounine avait propagées depuis 1866. ${ }^{2}$

Ce célèbre "Programme de la Commune", lu par Vallès à la séance du 18 avril, fut intégralement rédigé par Delescluze. C'était évidemment un programme plutôt proudhonien, voire bakouninien que jacobin blanquiste. Après en avoir entendu la lecture, Paul Rastoul s'écria: «Ceci est l'oraison funèbre du jacobinisme, prononcée par un de ses chefs." ${ }^{3}$ Il faut remarquer, d'ailleurs, que la Déclaration n'était pas uune simple concession des Parisiens à la province, mais l'expression d'un courant politique enraciné. $)^{4}$

Le 5 avril 1871, dans une lettre à Ozerov, Bakounine analyse les événements qui se déroulent en France:

«Selon toutes probabilités, écrit-il, les Parisiens seront vaincus, mais ils ne périront pas inutilement, ils auront fait beaucoup de besogne. Qu'ils fassent sauter Paris même, s'il le faut! Malheureusement, les villes de province comme Lyon, Marseille et autres, ne montrent pas plus de vaillance qu'au commencement, s'il faut en croire les nouvelles qui me parviennent. Les anciens jacobins, les Delescluze, les Flourens, les Pyat et Blanqui lui-même, devenus

1 Gustave Lefrançais, Etude sur le mouvement communaliste à Paris en 1871, Neuchâtel 1871, p. 197.

2 Naturellement, il ne faut pas trop généraliser l'extension de la Commune. Il faut bien distinguer la notion d'une "autonomie municipale" des fédéralistes républicains ou des proudhoniens modérés, le communalisme d'un Lefrançais, surtout la "Commune révolutionnaire» des blanquistes et néo-jacobins, de celle de Bakounine - la Commune comme base d'un fédéralisme anti-autoritaire et antiétatique.

3 Georges Bourgin et Gabriel Henriot, Procès-verbaux de la Commune de 1871, Edition critique, t. I (mars-avril 1871), Paris 1924, p. 274.

4 J. Gaillard, op. cit., p. 155. 
membres de la Commune ne laissent pas de me donner de nouvelles inquiétudes. Je crains qu'ils n'entraînent et ne maintiennent le mouvement dans l'ancienne voie de coupe-tête et d'économie de finances. Alors tout sera perdu. 〈Une et indivisible> va annuler tout et surtout elle se perdra elle-même. Ce qui donne de la valeur à cette révolution, c'est précisément qu'elle a été faite par la classe ouvrière. Voilà ce que peut produire une organisation. Durant le siège de Paris, nos amis avaient eu le temps de s'organiser; ils surent créer une force formidable, tandis que nos Lyonnais et nos Marseillais demeurèrent bredouilles. Les hommes de talent et d'énergie se concentrent en trop grand nombre à Paris; je crains qu'ils ne s'entravent même les uns les autres. Mais alors, en province, les hommes manquent totalement. S'il n'est pas encore trop tard, il faut insister pour envoyer en province un nombre de délégués, de véritables révolutionnaires. $»^{{ }^{1}}$

Bakounine partit le 25 avril de Locarno et se fixa à Sonvilliers, chez Schwitzguébel. Là, on discutait des mesures multiples pour une action. Ozerov tentait d'organiser une colonne armée pour aller, avec les Jurassiens, semer la révolte en France. On envisageait la proclamation d'une Commune à Besançon... Mais pour tout cela, c'était trop tard. Bientôt, il ne s'agissait que d'actions de sauvetage des victimes de la Semaine sanglante.

Pendant la Commune, Arman Ross, ${ }^{2}$ le révolutionnaire russe alors le plus proche de Bakounine, se trouvait à Paris et y était en contact avec Varlin. De Genève, il avait apporté une lettre d'Ozerov destinée à Dombrowski, qu'Ozerov connaissait de Russie et de Paris. Le 5 avril, Bakounine expédia à Ozerov une lettre pour Varlin qu'il avait écrite la veille. Cette lettre est perdue et on ignore si Varlin l'a reçue. De Paris, Bakounine recevait des lettres perspicaces de Ross disant qu'il était très pessimiste et estimait que cette révolution mal organisée aurait une conclusion mauvaise. Ross parvint à s'échapper de Paris; durant le massacre, il s'y cacha dans la clinique de Vyrubov. ${ }^{3}$ Le 10 juin, Bakounine informe James Guillaume qu'il a reçu une lettre de Ross et qu'il l'engage beaucoup «à écire son journal le plus détaillé et le plus

1 Pis'ma, op. cit., p. 316.

2 Michail Petrovič Sažin qui, depuis 1870, se faisait appeler Arman Ross, était né en 1845. En 1865, il fut poursuivi pour avoir pris part à l'impression de l'ouvrage, interdit en Russie, de Ludwig Büchner, Force et matière, et déporté en 1868 par mesure administrative. L'été 1869 , il s'évada et se réfugia en Amérique. Il arriva fin mai 1870 à Genève et fit connaissance aussitôt avec Bakounine. Jusqu'à 1874, il resta intimement lié avec ce dernier.

${ }_{3}$ M. P. Sažin, Vospominanija, Moscou 1925, pp. 75-80. 
sévèrement véridique. Nous le traduirons, d'abord pour les amis intimes, parce que toute vérité n'est pas bonne à dire en public: nous ne devons pas diminuer le prestige de ce fait immense, la Commune, et nous devons défendre à outrance, dans ce moment, même les jacobins qui sont morts pour elle.» Malheureusement, Ross n'a pas suivi ce conseil.

Quant à Bakounine, cinq jours auparavant il avait déjà commencé sa défense de la Commune, un de ses écrits le plus fameux et le plus répandu, mais qui, lui aussi, ne fut publié qu'à titre posthume. " $\mathrm{Je}$ suis, écrit-il, un partisan de la Commune de Paris, [...] surtout parce qu'elle a été une négation audacieuse, bien prononcée, de l'Etat.»Dans sa terminologie anarchiste de l'époque, il voyait dans la Commune, ce "fait historique immense», une première manifestation du socialisme révolutionnaire, tout à fait hostile à l'Etat. "Les communistes [Bakounine entend par là les socialistes d'Etat et les marxiens] croient devoir organiser les forces ouvrières pour s'emparer de la puissance politique des Etats. Les socialistes révolutionnaires s'organisent en vue de la destruction ou, si l'on veut un mot plus poli, en vue de la liquidation des Etats.» Ceux-ci ont beaucoup plus de confiance dans les aspirations instinctives des masses populaires que dans l'intelligence profonde de tous les docteurs et tuteurs de l'humanité. Ils pensent que celle-ci s'est laissée trop longtemps gouverner et que «la source de ces malheurs ne réside pas dans telle ou telle forme de gouvernement, mais dans le principe et dans le fait même du gouvernement quel qu'il soit.» C'est là la contradiction, devenue déjà historique, qui existe entre le communisme scientifique d'un côté, et le proudhonisme largement développé et poussé jusqu'à ses dernières conséquences de l'autre. ${ }^{3}$

Après le Congrès de La Haye, Bakounine, polémiquant contre Marx, revient sur le caractère anti-marxiste de la Commune. Ce qui en constitue l'importance, ce ne sont pas les bien faibles essais qu'elle a eu la possibilité et le temps de faire, c'est la lumière vive qu'elle a jetée sur la vraie nature et sur le but de la révolution, dont le trait principal est la révolte de la Commune et des associations ouvrières contre l'Etat. «L'effet en fut si formidable partout, que les marxiens eux-mêmes, dont toutes les idées avaient été renversées par cette insurrection, se virent obligés de tirer devant elle leur chapeau. Ils firent plus: à l'inverse de la plus simple logique et de leurs sentiments véritables, ils proclamèrent

1 Guillaume, op. cit., p. 156.

2 Publié pour la première fois, dans une version très libre, par Elisée Reclus en 1878 , et réimprimé maintes fois après sous le titre La Commune de Paris et la notion d'Etat.

3 Bakounine, Guvres, t. IV, pp. 250-253. 
que son programme et son but étaient les leurs. Ce fut un travestissement vraiment bouffon, mais forcé. Ils avaient dû le faire, sous peine de se voir débordés et abandonnés de tous, tellement la passion que cette révolution avait provoquée en tout le monde avait été puissante.»1 Bakounine vise ici, bien entendu, La Guerre civile en France.

Même si l'on tient compte du fait que tous les écrits contemporains cherchaient à dégager le sens théorique plutôt qu'à faire l'historique des phénomènes concrets et complexes, souvent contradictoires de la Commune, on peut dire en résumé que la Commune, du point de vue de la théorie socialiste, puisait ses tendances fondamentales plutôt dans une collectivisation, dans le sens de Bakounine ou de Varlin, que dans une étatisation de la production. Il s'agissait, au fond, d'un communalisme fédéraliste. On peut bien souscrire aux conclusions de Marx: «Non seulement l'administration municipale, mais toute l'initiative jusqu'alors exercée par l'Etat fut remise aux mains de la Commune. [...] La Commune de Paris devait, bien entendu, servir de modèle à tous les grands centres industriels de France. Le régime de la Commune une fois établi à Paris et dans les centres secondaires, l'ancien gouvernement centralisé aurait, dans les provinces aussi, dû faire place au gouvernement des producteurs par eux-mêmes.. ${ }^{2}$

Et même si l'on ne va pas aussi loin que Marx, qui a vu dans la Commune une tentative d'extirper l'Etat, on pourrait en résumer l'expérience et toute l'importance politique dans les mots d'un de ses membres, Lefrançais: "Le caractère principal, en effet, du mouvement du 18 mars, c'est que ce mouvement aura été le point de départ d'une rupture complète et sans retour possible d'avec les divers partis politiques qui, à différents titres, avaient eu jusqu'alors la prétention de représenter la révolution.»" Déjà en 1871 , le théoricien du "communalisme» affirma la tendance anti-étatique de la Commune en écrivant que celle-ci «n'avait pas seulement pour but de décentraliser le pouvoir, mais de faire disparaître le pouvoir lui-même. $\|^{4}$

Il est évident que tout cela s'accorde difficilement avec le système de Marx. Il me semble que c'est travestir l'œuvre et la pensée de ce dernier que de vouloir intégrer les éléments anti-étatiques de La Guerre civile dans le socialisme scientifique, voire dans la théorie d'un processus dialectique où, le moment venu, la classe ouvrière organisée en parti politique doit conquérir l'Etat afin de concentrer les moyens de pro-

1 Archives Bakounine, t. II, p. 166.

2 La Guerre civile, op. cit., pp. 42-43.

3 Gustave Lefrançais, "Communalisme», in: La Commune. Revue socialiste, Ie année, n०3, juin 1874, p. 9.

4 Lefrançais, Etude, op. cit., p. 368 
duction dans les mains d'un système politique qui prend le nom de «dictature du prolétariat». Dans La Guerre civile, d'ailleurs, ces mots ne figurent pas. C'est Engels, on le sait, qui en 1891 écrit: «Regardez la Commune de Paris. C'était la dictature du prolétariat.» Belle expression mais qui ne signifie rien. ${ }^{1}$

La Commune ne veut pas attendre que l'Etat dépérisse; son abolition ne sera pas pour elle le résultat final et inéluctable d'un processus historique, d'une phase suprême atteinte par la société et déterminée par une forme de production supérieure. Aucune des conditions définies antérieurement et postérieurement par Marx comme prélude à la conquête de l'Etat et à sa suppression subséquente n'a donc été remplie par la Commune. Des marxistes de toutes tendances, ayant de la probité intellectuelle et le respect de l'histoire, tels qu'Eduard Bernstein, Franz Mehring, Arthur Rosenberg, Karl Korsch, ont reconnu ce fait. ${ }^{2}$

En outre, d'abord la victoire de Bismarck n'inspirait pas à Marx et Engels le moindre regret. Le 20 juillet 1870, Marx écrit à Engels: «Les Français ont besoin d'être rossés. Si les Prussiens sont victorieux, la centralisation du pouvoir de l'Etat sera utile à la centralisation de la classe ouvrière allemande. La prépondérance allemande, en outre, transférera le centre de gravité du mouvement ouvrier européen de France en Allemagne; et il suffit de comparer le mouvement dans les deux pays, depuis 1866 jusqu'à présent, pour voir que la classe ouvrière allemande est supérieure à la française tant au point de vue théorique qu'à celui de l'organisation. La prépondérance, sur le plan mondial, du

1 Ou plutôt le contraire, comme le dit très bien cette thèse: «Le mot d'Engels: <Regardez la Commune de Paris. C'était la dictature du prolétariat〉, doit être pris au sérieux, comme base pour faire voir ce que n'est pas la dictature du prolétariat en tant que régime politique (les diverses modalités de dictature sur le prolétariat, en son nom)." (Internationale situationniste, Paris, no 12, 1969)

2 Franz Mehring, le biographe de Marx, a écrit à propos de La Guerre civile: "Aussi brillants que fussent ces développements pris en détail, ils n'en étaient pas moins en contradiction certaine avec les idées que Marx et Engels avaient représentées depuis un quart de siècle et qu'ils avaient déjà rendues publiques dans le Manifeste communiste. D'après leur conception, il y avait, bien entendu, parmi les conséquences ultimes de la future révolution prolétarienne, la dissolution de l'organisation politique connue sous le nom d'Etat, mais il ne s'agissait cependant que d'une <dissolution progressive>. [...] Pour atteindre ce but et les autres objectifs encore plus importants de la future révolution sociale, Marx et Engels insistaient en même temps sur la nécessité pour la classe ouvrière de <s'emparer du pouvoir organisé de l'Etat $>[\ldots]$ Cette conception formulée dans le Manifeste communiste, ne pouvait s'accorder avec les louanges que l'Adresse du Conseil général de l'Internationale décernait à la Commune de Paris <pour avoir commencé à radicalement extirper l'Etat parasitaire >." (Franz Mehring, Karl Marx. Geschichte seines Lebens, s.1. 1923, p. 460) 
prolétariat allemand sur le prolétariat français serait en même temps la prépondérance de notre théorie sur celle de Proudhon.»1

Quelques semaines plus tard, le 15 août, Engels, à son tour, écrit à Marx: «Comme en 1866, Bismarck fait encore une partie de notre besogne [...].»" Naturellement, Engels a en vue la centralisation économique et politique de l'Allemagne, condition préalable, d'après la conception de Marx, pour le développement du socialisme. Il faut rappeler, d'ailleurs, que, dès 1865, pour Marx et Engels, l'armée prussienne avait une tâche nationale et progressive à accomplir. Le Junker prussien représentait une force progressive, comme l'armée prussienne, travaillant à l'unité nationale allemande. "Il est évident, écrit Marx en 1866, que tout ce qui centralise la bourgeoisie est favorable aux ouvriers. $\|^{3}$

Trois mois après son écrit sur la Commune, Marx s'efforça de transformer l'Internationale, jusqu'alors confédération de fédérations autonomes, en centrale de partis, rendant du même coup obligatoires la constitution du prolétariat en parti politique et son action politique pour la conquête de l'Etat. On est bien loin des phrases sur l'abolition de l'Etat qu'on trouve dans La Guerre civile.

Marx considérait la guerre franco-allemande du point de vue de la politique européenne. Dans la première Adresse du Conseil général, en date du 23 juillet 1870, il estime encore que, du côté allemand, la guerre est une guerre de défense. ${ }^{4}$ A l'arrière-plan, «la sinistre figure de la Russie est à l'affût». Lorsque les desseins impérialistes de la Prusse devinrent évidents, Marx fut convaincu, cette fois, qu'il fallait que l'Angleterre déclarât la guerre à la Prusse et à son allié, la Russie. Le moment de la faire, pensait-il, s'était rencontré lorsque la Russie avait répudié le Traité de Paris. ${ }^{5}$ Une résolution allant dans ce sens, rédigée

1 Marx et Engels, Werke, t. 33, p. 5.

2 Ibid., p. 40.

3 Lettre à Engels, 27 juillet 1866, Werke, t. 31, Berlin 1965, p. 243.

4 Le 15 août 1870, écrivant à Marx, Engels répète ces propos en ajoutant que la guerre de défense "n'exclut pas au demeurant l'offensive jusqu'à la paix" (Werke, t. 33, p. 40).

5 Dans sa lettre du 13 décembre 1870 à Kugelmann, Marx écrit encore: "La clé de la question est là: par le traité de Paris de 1856, l'Angleterre s'est elle-même désarmée. [...] Messieurs les Russes et Messieurs les Prussiens comptent sans leur hôte, s'ils se figurent que l'influence de la reine qui, par family interest, est liée à la Prusse, et l'imbécillité bourgeoise d'un Gladstone empêcheront, au moment décisif, John Bull de jeter par-dessus bord l'obstacle qu'il s'est luimême créé. Il peut toujours, en quelques semaines, donner le coup de grâce au commerce maritime de la Russie et de la Prusse.) (Karl Marx, Lettres à Kugelmann (1862-1874), Paris 1930, p. 153) 
par Engels et approuvée par Marx, fut acceptée par le Conseil général. ${ }^{1}$ Impossible de résumer ici - et en général - les variations étranges des opinions de Marx et Engels sur la guerre et les instructions qu'ils donnèrent par l'intermédiaire de l'Internationale aux ouvriers français, instructions qui, d'ailleurs, n'eurent pas la moindre influence. ${ }^{2}$

Tandis que Marx, russophobe, avait un penchant pour l'unification et la centralisation de l'Allemagne découlant de sa conception de la conquête de l'Etat, Bakounine voyait dans cette même centralisation et dans l'unité de l'Allemagne une tendance dangereuse à la "prussogermanisation" de l'Europe. Après l'écrasement de la Commune, Bakounine regardait l'Etat allemand, créé et unifié par les forces conservatrices, comme le plus grand danger qui menaçait l'Europe. Quant à la Commune, il la nomme en 1872 encore une fois "cette négation désormais historique de l'Etat». ${ }^{3}$

1 Il y était dit: "1) Que le mouvement ouvrier, pour le soutien de la République française, aurait dû en premier concentrer ses efforts pour obliger le gouvernement britannique à reconnaître la République; [..] 3) Que l'Angleterre non seulement demeure incapable d'intervenir efficacement dans les affaires continentales, mais qu'elle ne peut même pas se protéger contre le despotisme militaire du continent, tant qu'elle n'aura pas reconquis la faculté de disposer de sa véritable force militaire, sa flotte de guerre. Elle ne le peut qu'en dénonçant la Déclaration de Paris." (Minutes, t. IV, pp. 112-113) Le 14 mars, Marx parla de nouveau dans ce sens (ibid., pp. 153-154).

2 Une des choses la plus curieuse et un peu obscure est le fait relaté par l'historien marxiste Boris Nikolaevskij: «Par l'intermédiaire de Lafargue, Engels fit parvenir au secrétaire de Gambetta un mémorandum comprenant des plans soigneusement établis pour débloquer Paris." (Boris Nicolaievski et Otto Maenchen-Helfen, La vie de Karl Marx, Paris 1970, p. 362) Ce fait se trouve aussi mentionné dans la biographie d'Engels par Gustav Mayer qui cite à propos de cette affaire le document suivant trouvé dans les papiers d'Engels: «Le paquet $\mathrm{n}^{\circ} 38$ a été détruit par décision commune. A. Bebel, Eduard Bernstein, Londres, 24 juillet 1896." Selon Mayer, ces papiers auraient été détruits parce qu'on craignait que le Parti socialiste ne fût accusé ultérieurement de haute trahison (G. Mayer, Friedrich Engels, t. II, La Haye 1934, pp. 197, 544-545).

3 Archives Bakounine, t. II, p. 213. 